\title{
Therapeutic Treatment of Hepatic Colorectal Metastases
}

\author{
a report by \\ Jason K Sicklick and Timothy M Pawlik
}

Department of Surgery, Division of Surgical Oncology, The Johns Hopkins University School of Medicine, Baltimore

DOI: 10.17925/EOH.2007.0.2.63

In Western countries, colorectal cancer is the fourth leading cause of cancer. In the US it is the second most common cause of cancer-related mortality. Unfortunately, about $35-55 \%$ of patients with colorectal cancer develop hepatic colorectal metastases. At present, surgical resection of these lesions represents the main therapeutic option and is the only chance for cure and long-term survival. In patients with extensive metastatic disease who are not candidates for resection only, radiofrequency ablation (RFA) can be combined with hepatectomy. While the principal goal of hepatic resection is to resect all metastases with negative histological margins, preservation of adequate liver parenchyma must be considered. The utilisation of portal vein embolisation (PVE) and neoadjuvant chemotherapy may lead to increased candidacy for surgical treatment of such colorectal metastases. Although a fraction of patients develop recurrent disease after hepatic resection, newer and more biologically active chemotherapeutic agents are available that may prolong both diseasefree and overall survival. The current therapeutic options for hepatic colorectal metastases necessitate a multidisciplinary approach that involves surgeons, oncologists and radiologists in order to increase the number of patients who are candidates for the surgical treatment of colorectal cancer liver metastasis.

In the US there are approximately 150,000 new cases of colorectal cancer annually, accounting for more than 55,000 cancer-related deaths. ${ }^{1}$ Unfortunately, approximately $50 \%$ of these patients will develop hepatic colorectal metastases. ${ }^{2}$ Of these patients, $15-25 \%$ present with synchronous hepatic lesions, ${ }^{3-5}$ while another $20-25 \%$ will develop metachronous liver disease. ${ }^{6-8}$ Furthermore, in one-fifth of these patients the liver is the only site of metastatic disease. ${ }^{9}$ As a result, there are approximately 10,000-15,000 patients each year who are candidates for surgical resection of their hepatic colorectal metastases. ${ }^{8}$

Surgical resection of hepatic metastases remains the only therapeutic option with the potential for long-term cure. ${ }^{10}$ Long-term survival and potential cure following surgical resection for hepatic colorectal metastases have been demonstrated in numerous studies. The overall five-year survival rate reported after hepatectomy with curative intent ranges from 35 to $58 \% .10-19$ These results are expected to improve even further with a multidisciplinary approach that includes newer chemotherapy regimens. ${ }^{20}$ As such, resection of colorectal liver metastasis should be considered standard practice. Furthermore, many traditional factors that were previously considered absolute contraindications to hepatic resection have recently been called into question. For example, even very well selected patients with extrahepatic metastatic disease may now be considered potential candidates for hepatic resection. ${ }^{21,22}$ Herein, we review the issues relating to the therapeutic management of patients with colorectal liver metastases.
Pre-operative Evaluation

\section{Patient Selection}

Peri-operative mortality following hepatectomy has decreased from $20 \%$ pre-1980 to less than $5 \%$ post-1980.23-29 A key component in decreasing the risk of post-operative complications is the appropriate selection of surgical candidates. Several factors must be considered in the decision to offer major liver resection as a surgical therapy. Co-morbidites and age are both factors, although age itself is not an independent predictor of increased operative risk. ${ }^{30,31}$ Surrogate markers of general physiological fitness, such as the American Society of Anesthesiology (ASA) score and the pre-operative acute physiology and chronic health evaluation (APACHE) score, are better indicators of post-operative complications

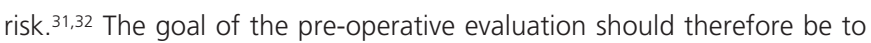
identify those patients who are at a high operative risk such that their co-morbidities can be appropriately addressed in the pre-operative setting, thus reducing the risk of post-operative complications.

\section{Imaging}

Every patient being considered for resection of hepatic colorectal metastases should have cross-sectional imaging performed to evaluate the extent of intrahepatic disease and to exclude extrahepatic metastasis. In general, pre-operative computed tomography (CT) is the imaging modality of choice, although some centres favour magnetic resonance imaging (MRI) to evaluate liver-based disease. Pre-operative imaging should be used to define the number of liver lesions and their distribution, as well as their proximity to major biliary and vascular structures. The lungs should also be evaluated pre-operatively with either a chest radiograph or a chest $\mathrm{CT}$ to rule out pulmonary metastasis. ${ }^{33,34} \mathrm{~A}$ mandatory colonoscopy within one year is also required to exclude either anastomotic recurrence or a metachronous colorectal tumour. More recently, positron emission tomography (PET) with the radiolabelled glucose analogue [18F] fluoro-2-deoxy-D-glucose (FDG) has been used as a sensitive diagnostic tool to image tumours based on

Jason K Sicklick is a General Surgery Resident at the Johns Hopkins University School of Medicine. He will complete his residency in 2008 and then commence a fellowship in surgical oncology at Memorial Sloan-Kettering Cancer Center. Dr Sicklick received his medical degree from the University of California, Los Angeles School of Medicine in 2000.

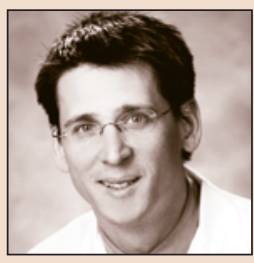

Timothy M Pawlik is an Assistant Professor in the Department of Surgery, Division of Surgical Oncology at the Johns Hopkins School of Medicine, Baltimore. His clinical interests include alimentary tract surgery, liver cancers, gallbladder and bile duct cancers, pancreas cancers and gastrointestinal cancers. His research interests focus on experimental therapeutics of hepatic malignancies, clinica trials in gastrointestinal malignancies and clinical ethics.

E: tpawlick1@jhmi.edu 


\section{Liver Cancer}

increased uptake of glucose by tumour cells. Retrospective studies have suggested that the ability of FDG-PET to detect extrahepatic disease may improve patient selection for surgery and thereby result in better survival rates following resection. ${ }^{35}$ Furthermore, PET has recently been combined with $\mathrm{CT}$ (PET/CT) in an attempt to improve identification of extra- and intrahepatic lesions. ${ }^{36,37}$ Data suggest that PET/CT provides the most accurate pre-operative imaging for staging purposes. As a result, some investigators ${ }^{36}$ advocate that PET/CT scans be routinely performed on all patients being evaluated for liver resection for metastatic colorectal cancer.

\section{Tumour and Underlying Liver-related Factors}

Traditionally, liver resection for colorectal metastases was deemed inappropriate in patients who had any one of a strict group of criteria, including: four or more metastatic lesions, hilar adenopathy, metastases within $1 \mathrm{~cm}$ of major vessels (i.e. inferior vena cava or main hepatic veins) or any evidence of extrahepatic disease. The clinical and methodological basis of these studies has been criticised over the years. Methodologically, many of these studies failed to control for many factors and patient selection was varied and non-standardised. Clinically, the data are problematic because many of the studies were conducted prior to the utilisation of more effective, new-era chemotherapy agents. In addition, many of these studies were carried out before the introduction and widespread use of techniques such as RFA or PVE. More recent studies have suggested that patients with 'traditional' adverse clinicopathological factors can achieve long-term survival following hepatic resection and, therefore, should not be excluded from surgical consideration. This has led to a shift in the definition of the resectability of hepatic liver metastases. Newer criteria rely more on whether macroscopically complete (R1) and microscopically complete (RO) resection of the liver lesions can be performed. Hepatic colorectal metastases should be defined as resectable when it is anticipated that disease can be completely resected (i.e. Ro resection), two adjacent liver segments can be spared, adequate vascular inflow and outflow and biliary drainage can be preserved and the volume of the liver remaining after resection (i.e. the 'future liver remnant') will be adequate (in general, at least $20-30 \%$ of the total estimated liver volume). ${ }^{38,39}$ This new definition of resectability represents a clear paradigm shift. Rather than resectability being defined by what is removed, decisions regarding resectability now focus on what will remain.

In general, $20 \%$ of the total liver volume appears to be the minimum safe volume that can be left following liver resection in patients with normal hepatic function. However, the cut-off threshold may need to be increased in those patients with underlying liver injury secondary to steatosis or steatohepatitis (30\%) or cirrhosis (40\%). CT or MRI can now provide an accurate, reproducible method for pre-operatively measuring the volume of the future liver remnant. ${ }^{39}$ For those patients who fail to have compensatory hepatic hypertrophy as a result of tumour growth, or who have a future liver remnant of less than 20\%, PVE should be considered to induce hypertrophy of the contralateral liver lobe. ${ }^{40}$ The concept of PVE emerged from the recognition that portal invasion by tumour leads to ipsilateral hepatic lobar atrophy and contralateral lobar hypertrophy. In most cases, PVE involves the purcutaneous cannulation of the ipsilateral portal vein under direct fluoroscopic control followed by portography and selected PVE using polyvinyl alcohol and microcoils. PVE is safe, with a complication rate of less than $5 \%$, causes little peri-portal reaction and generates durable portal vein occlusion, especially when used in combination with coils. ${ }^{41}$ PVE has been shown both to increase the size of the future liver remnant and to decrease the incidence of post-operative complications in patients who would otherwise have had an inadequate liver remnant. Thus, the selective use of PVE may enable safe and potentially curative extended hepatectomy in a subset of patients with extensive hepatic colorectal metastases who would otherwise have been marginal candidates for resection. Historically, the presence of extrahepatic metastatic disease was considered an absolute contraindication to hepatic resection for colorectal metastases because early reports found that no patients had survived beyond five years. ${ }^{15}$ However, with more effective systemic chemotherapeutic agents and improved imaging modalities that can more accurately identify the extent of extrahepatic disease, some clinical investigators have begun to advocate hepatic resection in patients with extrahepatic disease. In one study, Elias et al.22 reported that the fiveyear survival rate following hepatectomy for colorectal liver metastases with simultaneous resection of extrahepatic disease with curative intent was $29 \%$. Thus, extrahepatic disease should not be considered an absolute contraindication to hepatic resection. However, these patients need to be exceptionally well selected. Only very carefully selected patients who have been treated with systemic chemotherapy and have demonstrated documented stable and/or responsive disease should be considered operative candidates. In addition, only those patients with a complete (RO) surgical extirpation of all (i.e. both pre- and post-chemotherapy) sites should be offered resection of their intra- and extrahepatic disease. The treatment of these patients involves complex clinical decision-making, and should be carried out in a multidisciplinary setting.

\section{Operative Considerations}

\section{Non-anatomical versus Anatomical Resection and Extent of Surgical Margin}

The basic tenets for the resection of hepatic colorectal metastasis include a clear pathological surgical margin and the preservation of sufficient functional hepatic parenchyma. There remains some controversy surrounding whether one should perform an anatomical versus a nonanatomical resection for hepatic colorectal metastases. ${ }^{42,43}$ Unlike hepatocellular carcinoma, the benefits of anatomical resection in patients with colorectal liver metastasis have not been demonstrated. In fact, the use of non-anatomical hepatic resection has been justified based on several important pathological factors that differ between hepatocellular carcinoma versus metastatic colorectal cancer. Specifically, metastatic colorectal cancer tends to be characterised by histologically well-circumscribed lesions, ${ }^{44}$ uncommon satellitosis and Glisson sheath extension, 7,45 and rare micrometastases in the liver parenchyma surrounding colorectal liver metastasis (and, if present, they are usually found in close proximity to the hepatic metastasis). ${ }^{45}$ Several studies have reported similar rates of positive surgical margins and five-year survival for patients treated with nonanatomical versus anatomical resection. ${ }^{45-47}$ Thus, rather than dogmatically adhering to an anatomical versus a non-anatomical approach, the surgeon's goal should be to resect all metastases with negative histological margins while preserving sufficient functional hepatic parenchyma. Surgical margin status is another controversial factor that has been evaluated for its influence on long-term survival after resection of colorectal liver metastases. Based on current data, however, a surgical margin of at least $1 \mathrm{~mm}$ appears not to place the patient at any increased risk of margin-related recurrences or worse prognosis. ${ }^{48}$ Pawlik et al. ${ }^{48}$ noted that the width of a negative surgical margin did not affect survival, recurrence risk or site of recurrence following hepatic resection of colorectal metastases. Therefore, a predicted margin of less than $1 \mathrm{~cm}$ after resection of hepatic colorectal metastases should not be used as an exclusion criterion for resection. 


\section{Hilar Lymph Node Metastasis}

Hilar lymph node metastasis is recognised as an important predictor of prognosis. While some authors ${ }^{49}$ recommend that lymphadenectomy should always be performed at the time of hepatic resection, the routine addition of this procedure to hepatectomy for metastatic colorectal liver disease remains controversial. However, the presence of hepatic lymph node metastasis should not be used as an exclusionary criterion for hepatic resection. Although some investigators have reported very poor outcome in patients with positive hepatic lymph nodes, ${ }^{50-52}$ others have reported favourable survival in patients with hilar nodal metastases and have concluded that this patient population may still benefit from hepatic resection. ${ }^{42,53,54}$

\section{Radiofrequency Ablation}

RFA has become a widely used ablative technique for liver tumours. RFA involves the localised application of thermal energy to destroy tumour cells. Specifically, alternating electric current in the range of RF waves $(460 \mathrm{kHz})$ is applied from an RF generator through a needle electrode placed directly into the tumour. ${ }^{55}$ As the temperature within the tissue becomes elevated above $60^{\circ} \mathrm{C}$, cells are denatured and die, and a zone of necrosis surrounding the electrode develops. ${ }^{56}$ Tumours less than $2.5 \mathrm{~cm}$ in greatest diameter can usually be ablated with a single central placement of a needle electrode array of $3.5-4.0 \mathrm{~cm} .{ }^{57}$ Larger tumours require multiple deployments of the needle electrode. Treatment should be planned such that the zones of coagulative necrosis overlap to ensure complete destruction of the tumour. ${ }^{57}$ Ideally, a thermal zone of ablation measuring $1 \mathrm{~cm}$ wide around the tumour should be created to ensure destruction of the tumour as well as a rim of non-malignant tissue. Tumour abutting major hepatic or portal vein branches can be safely ablated as the blood within these vessels acts as a heat sink to protect the vascular endothelium from thermal injury, while allowing complete coagulative necrosis of the tissue surrounding the vessel wall. ${ }^{77,58}$ RFA should not, however, be applied to lesions near the hilar plate; although the blood vessels can tolerate the heat generated from RFA, the large bile ducts in this area do not tolerate heat and RFA can induce serious damage to these structures. ${ }^{57}$ RFA can be performed percutaneously, laparoscopically or as open surgery. ${ }^{57,59}$ RFA should not be viewed as a replacement for resection, which remains the gold standard for the treatment of colorectal liver metastasis.11,57,60 In general, patients with resectable colorectal liver metastasis should undergo resection, not RFA. Rather, the main indication for RFA is in patients who do not meet the criteria for resectability, but are candidates for liver-directed therapy. RFA can also be combined with liver resection so that, following resection of the bulk of disease, smaller 'unresectable' metastases can be ablated. ${ }^{60}$

Survival following RFA is difficult to interpret, as many patients who undergo RFA are characterised by a number of poor prognostic factors (i.e. 'unresectable' disease, multiple tumours, bilobar location, etc.), making comparisons with patients who have undergone complete surgical resection difficult. In a recent study comparing recurrence and outcome following hepatic resection, RFA or combined resection and RFA for colorectal liver metastases, the authors ${ }^{11}$ reported a significantly worse disease-free and overall survival for patients treated with RFA. Data from a more recent study have suggested that significant selection bias may influence any analyses attempting to compare RFA alone, resection alone and RFA plus resection. ${ }^{61}$ Collectively, these data serve to highlight the need for a randomised trial comparing resection versus RFA before RFA can be accepted as standard therapy for patients with potentially resectable colorectal liver metastases.

\section{Chemotherapy}

\section{Systemic Therapy}

Conventional first-line chemotherapy regimens for metastatic colorectal disease contain fluorouracil (5-FU) and leucovorin, which potentiates 5-FU's inhibition of DNA and RNA synthesis in malignant cells. ${ }^{62}$ Using a bolus administration regimen, patients treated with 5 -FU and leucovorin have reported response rates ranging from 20 to $30 \%$ and a median survival of 11.5 months. ${ }^{63-67}$ Over the past decade, additional chemotherapeutic and biologic agents have been found that have increased activity against colorectal cancer. For example, irinotecan is a topoisomerase I inhibitor initially introduced as monotherapy for patients with metastatic colorectal cancer refractory to 5-FU. ${ }^{68-70}$ Saltz and colleagues ${ }^{64}$ subsequently showed that combining irinotecan with bolus 5-FU/leucovorin (IFL regimen) resulted in a higher response rate (39\%), longer progression-free survival time and longer overall survival time (14.8 months) compared with bolus 5-FU/leucovorin alone. Concurrently, Douillard and colleagues ${ }^{71}$ showed that irinotecan in combination with continuous infusion 5-FU/leucovorin (FOLFIRI regimen) produced better response rates and longer progressionfree and overall survival compared with 5-FU/leucovorin alone. Oxaliplatin, a cisplatin derivative with a similar mechanism of action to other platinum compounds, has also demonstrated significant activity in treating colorectal metastasis. ${ }^{72,73}$ Recently, the combination of oxaliplatin and infusional 5-FU/leucovorin (FOLFOX regimen) was found to be less toxic and more efficacious than the FOLFIRI regimen. ${ }^{62}$ Goldberg et al. ${ }^{62}$ reported that FOLFOX produced a higher response rate (45\%) and longer median survival (19.5 months) than IFL. In addition to novel chemotherapeutic agents, new molecular targeted therapeutic approaches have been developed. Bevacizumab, a monoclonal antibody to vascular endothelial growth factor A (VEGF-A) that inhibits angiogenesis, has been studied in phase II and III trials in patients with metastatic colorectal cancer. ${ }^{74,75}$ The addition of bevacizumab to cytotoxic chemotherapy has been shown to improve response rates and progression-free survival. ${ }^{74,75}$ The epidermal growth factor receptor (EGFR) signalling pathway has also been targeted by new biologic agents aimed at blocking the downstream signalling cascade that leads to tumour-cell proliferation, migration and inhibition of apoptosis. ${ }^{76}$ Cetuximab, an anti-EGFR monoclonal antibody, has been shown to have cytostatic activity as a single agent, but the combination of cetuximab and cytotoxic chemotherapy resulted in a synergistic antitumour response. ${ }^{77-80}$ Cetuximab is now approved as second-line therapy for patients with metastatic colorectal cancer. The role of adjuvant chemotherapy after potentially curative resection of liver metastases is uncertain. Given that the majority of patients with hepatic metastasis experience recurrence following resection, undetectable disease probably exists at the time of surgery. Systemic treatment of this subclinical disease with adjuvant chemotherapy is therefore rational.

\section{Neoadjuvant Chemotherapy for Resectable Metastases}

There is also increasing interest in using pre-operative chemotherapy for patients who present with resectable hepatic colorectal liver metastases. The rationale for this policy has been supported by the better prognosis obtained with neoadjuvant chemotherapy and surgery compared with immediate surgery in patients with multinodular colorectal liver metastases. ${ }^{81,82}$ Pre-operative systemic chemotherapy may also be associated with a lower rate $(3 \%)$ of positive surgical margins compared with immediate resection (9-19\%).83,84 A trial of chemotherapy also provides time to identify those patients who will develop progressive disease and therefore may not benefit from liver resection. ${ }^{81}$ In a study by Adam et al., ${ }^{81}$ patients who had tumour 


\section{Liver Cancer}

progression while receiving neoadjuvant chemotherapy had only an $8 \%$ overall five-year survival rate versus $37 \%$ for patients who had an objective tumour response to pre-operative chemotherapy. These data call into question the utility of hepatic resection in patients who fail to respond to neoadjuvant therapy, and may have important implications for the therapeutic strategy adopted by medical oncologists and surgeons. ${ }^{81}$ Whether routinely to give neoadjuvant chemotherapy for resectable disease is still somewhat controversial. This decision, therefore, should be individualised and based on the specific clinical situation. In patients who are chemo-naive with multiple metastases, or who have stage IV disease at presentation, neoadjuvant chemotherapy may be more appropriate. Neoadjuvant chemotherapy may also be warranted in those patients in whom a response would improve the outcome of the resection. In contrast, if a patient has co-morbidities, or there is concern about chemotherapy causing hepatoxicity in a patient who will require an extended resection, initial surgery would be more appropriate.

\section{Downstaging of Unresectable Metastases}

The improved efficacy of chemotherapy agents has not only increased patient survival in the palliative setting, but has also allowed a subset of previously unresectable patients to undergo liver surgery following tumour downstaging. ${ }^{85-87}$ Approximately $15-20 \%$ of patients with initially unresectable disease (defined as either the inability to resect all tumour while leaving $30 \%$ of the total liver volume or by concomitant presence of extrahepatic metastases) have significant tumour downstaging to the point at which the metastatic disease can ultimately be considered resectable. ${ }^{85,87}$ 'Rescue surgery' for previously unresectable colorectal liver metastases downstaged by chemotherapy can result in a five-year survival rate of $30-35 \% .{ }^{86}$

\section{Regional Therapy}

Regional chemotherapy has been proposed as treatment for unresectable colorectal disease or as adjuvant therapy following hepatic resection. ${ }^{88-92}$
Although several randomised controlled trials have demonstrated that hepatic arterial infusion (HAl) chemotherapy with fluorodeoxyuridine (FUDR) produced better response rates than systemic chemotherapy in patients with unresectable colorectal liver metastases, an improvement in survival was not detected. $88-90,92 \mathrm{HAl}$ as adjuvant therapy following hepatic resection has also not been shown to confer an improvement in long-term survival. ${ }^{91}$ Given that most patients are at risk of both intraand extrahepatic metastatic disease, and given that chemotherapy is now more efficacious, systemic chemotherapy should be favoured over regional approaches.

\section{Conclusion}

Liver resection currently represents the only potentially curative therapeutic option for hepatic colorectal metastasis, with five-year survival rates of 35-58\%. ${ }^{10-19}$ Recent improvements in whole-body and hepatic imaging have allowed for more accurate selection of patients with colorectal liver metastasis. Pre-operative imaging is now able to detect minimal burdens of metastatic disease that in the past would have been detected only after enlarging. Traditional clinicopathological factors, although helpful in stratifying patients with regard to prognosis, should not be used to exclude otherwise resectable patients from surgery. Modern surgical techniques have reduced peri-operative morbidity and mortality, while PVE and neoadjuvant chemotherapy have expanded the population of patients who are candidates for surgical treatment. In addition, more active systemic chemotherapy agents are being increasingly employed to consolidate therapy following surgery as the majority of patients who recur will have distant metastases as a component of their pattern of recurrence. Progress in the multidisciplinary approach to patients with colorectal liver metastasis has expanded the population of patients eligible for curative-intent surgery. As such, patients with metastatic colorectal cancer should be managed in a multimodality setting to offer these patients the best chance of long-term survival.
1. Jemal A, et al., CA Cancer J Clin, 2005;55:10-30

2. Steele G Jr, Ravikumar TS, Ann Surg, 1989;210:127-38.

3. Cady B, et al., Surg Gynecol Obstet, 1970;131:697-700.

4. Blumgart LH, Allison DJ, World J Surg, 1982;6:32-45.

5. Jatzko G, et al., Int J Colorectal Dis, 1991;6:111-14.

6. Finlay IG, McArdle CS, Br J Surg, 1986;73:732-5.

7. Scheele J, et al., World J Surg, 1995; 19:59-71.

8. Altendorf-Hofmann A, Scheele J, Surg Oncol Clin N Am, 2003;12:165-92, xi.

9. Scheele J, et al., Surgery, 1991;110:13-29.

10. Fong $Y$, et al., Ann Surg, 1999;230:309-18, discussion 18-21.

11. Abdalla EK, et al., Ann Surg, 2004;239:818-25, discussion 25-7.

12. Scheele J, et al., Br J Surg, 1990;77:1241-6.

13. Choti MA, et al., Ann Surg, 2002;235:759-66.

14. Hughes KS, et al., Dis Colon Rectum, 1988;31:1-4.

15. Adson MA, et al., Arch Surg, 1984;119:647-51.

16. Schlag P, et al., Eur J Surg Oncol, 1990;16:360-65.

17. Gayowski TJ, et al., Surgery, 1994;116:703-10, discussion 10-11.

18. Jenkins LT, et al., Am Surg, 1997;63:605-10.

19. Jamison RL, et al., Arch Surg, 1997;132:505-10, discussion 11

20. Bentrem DJ, et al., Annu Rev Med, 2005;56:139-56.

21. Pawlik TM, Choti MA, J Gastrointest Surg, 2007;11:1057-77.

22. Elias D, et al., Br J Surg, 2003;90:567-74.

23. Thompson HH, et al., Ann Surg, 1983;197:375-88

24. Fortner JG, et al., Ann Surg, 1978;188:363-71.

25. Tsao Jl, et al., Ann Surg, 1994;220:199-205.

26. Taylor M, et al., Am J Surg, 1997:173:467-71.

27. Bozzetti F, et al., Hepatogastroenterology, 1992;39:237-41.

28. Capussotti L, Polastri R, Hepatogastroenterology, 1998;45:184-90.

29. Sitzmann JV, Greene PS, Ann Surg, 1994;219:13-17.

30. Mentha G, et al., Br J Surg, 1992;79:557-9.

31. Belghiti J, et al., J Am Coll Surg, 2000;191:38-46.
32. Gagner M, et al., Surgery, 1991;110:487-92.

33. Kronawitter U, et al., Cancer, 1999;86:229-35.

34. Povoski SP, et al., Ann Surg Oncol, 1998;5:9-15.

35. Strasberg SM, et al., Ann Surg, 2001;233:293-9.

36. Selzner M, et al., Ann Surg, 2004;240:1027-34, discussion 35-6.

37. Hany TF, et al., Radiology, 2002;225:575-81.

38. Vauthey JN, et al., Ann Surg, 2004;239:722-30, discussion 30-32.

39. Abdalla EK, et al., Arch Surg, 2002;137:675-80, discussion 80-81.

40. Abdalla EK, et al., Br J Surg, 2001;88:165-75.

41. Duncan JR, et al., Radiology, 1999;210:467-77.

42. Yasui K, Shimizu Y, Int J Clin Oncol, 2005;10:86-96.

43. Yamamoto J, et al., Int J Clin Oncol, 2005;10:97-102.

44. Yamamoto J, et al., Ann Surg, 1995;221:74-8.

45. Kokudo N, et al., Arch Surg, 2002;137:833-40.

46. Zorzi D, et al., J Gastrointest Surg, 2006;10:86-94.

47. Kokudo N, et al., Am J Surg, 2001:181:153-9.

48. Pawlik TM, et al., Ann Surg, 2005;241:715-22, discussion 22-4.

49. Laurent C, et al., J Am Coll Surg, 2004;198:884-91.

50. Weber JC, et al., Am J Surg, 2001;182:81-8.

51. Hughes K, et al., Surg Clin North Am, 1989;69:339-59.

52. Barakos JA, et al., Gastrointest Radiol, 1990;15:93-101.

53. Nakamura S, et al., J Hepatobiliary Pancreat Surg, 1999;6:16-22.

54. Yasui K, et al., Ann Surg, 1997;226:582-6.

55. Rhim H, Dodd GD, 3rd, J Clin Ultrasound, 1999;27:221-9.

56. McGahan JP, et al., J Vasc Interv Radiol, 1992;3:291-7.

57. Curley SA, Izzo F, Cancer Metastasis Rev, 2004;23:165-82.

58. Curley SA, et al., Surg Endosc, 1997;11:729-33.

59. Machi J, et al., J Gastrointest Surg, 2001;5:477-89.

60. Pawlik TM, et al., Ann Surg Oncol, 2003;10:1059-69.

61. Gleisner AL, et al., Arch Surg, in press.

62. Goldberg RM, et al., J Clin Oncol, 2004;22:23-30.
63. Colucci G, et al., Anticancer Res, 1994;14:2157-62.

64. Saltz LB, et al., N Engl J Med, 2000;343:905-14.

65. Leichman CG, et al., J Clin Oncol, 1995;13:1303-11.

66. Buroker TR, et al., J Clin Oncol, 1994;12:14-20.

67. Advanced Colorectal Cancer Meta-analysis Project, I Clin Oncol, 1992;10:896-903.

68. Hsiang YH, et al., J Biol Chem, 1985;260:14873-8.

69. Rougier $P$, et al., Lancet, 1998;352:1407-12.

70. Cunningham D, et al., Lancet, 1998;352:1413-18.

71. Douillard JY, et al., Lancet, 2000;355:1041-7.

72. de Gramont A, et al., J Clin Oncol, 2000;18:2938-47.

73. Giacchetti S, et al., J Clin Oncol, 2000;18:136-47.

74. Hurwitz H, et al., N Engl J Med, 2004;350:2335-42.

75. Kabbinavar F, et al., J Clin Oncol, 2003;21:60-65.

76. Arteaga CL, I Clin Oncol, 2001;19:32S-40S.

77. Fan Z, et al., Cancer Res, 1993;53:4637-42.

78. Prewett MC, et al., Clin Cancer Res, 2002;8:994-1003.

79. Baselga J, et al., J Clin Oncol, 2000;18:904-14.

80. Ciardiello F, et al., Clin Cancer Res, 1999:5:909-16.

81. Adam R, et al., Ann Surg, 2004;240:1052-61, discussion 61-4.

82. Tanaka K, et al., Br J Surg, 2003;90:963-9.

83. Weber JC, et al., Ann Surg, 2002;236:560-63.

84. Parikh AA, et al., J Gastrointest Surg, 2003;7:1082-8.

85. Bismuth H, et al., Ann Surg, 1996;224:509-20, discussion 20-22.

86. Adam R, et al., Ann Surg, 2004;240:644-57, discussion 57-8.

87. Adam R, et al., Ann Surg Oncol, 2001;8:347-53.

88. Bonetti A, Lancet, 2003;361:358-9.

89. Rougier $\mathrm{P}$, et al., J Clin Oncol, 1992;10:1112-18.

90. Kemeny N, et al., Cancer, 1992;69:327-34.

91. Kemeny MM, et al., J Clin Oncol, 2002;20:1499-1505.

92. Meta-analysis Group in Cancer, J Natl Cancer Inst, 1996;88:252-8. 


\title{
When liver tumours predominate, consider SIF-Spheres microspheres
}

\section{Targeted therapy providing:}$$
\begin{aligned}
& \text { TIME Extends survival and to progression } \\
& \text { time to } \\
& \text { QOL } \text { Maintains quality }_{\text {of life, }}^{1,2}
\end{aligned}
$$$$
\text { SYNERGY Combined with }
$$$$
\text { ORP Improves response rates, }
$$$$
\text { even in refractory disease } \mathbf{e}^{1-3}-3
$$$$
\text { HOPE An }
$$$$
\text { - An alternate route to }
$$$$
\text { ablations-7 }
$$

SIR-Spheres microspheres (yttrium-90 resin microspheres) is indicated in the EU for the treatment of unresectable liver tumours.

Selective Internal Radiation Therapy (SIRT) using SIRSpheres is an effective and well-tolerated treatment for liver-only or liver-predominant tumours from primary disease and secondary metastases. ${ }^{1-9}$ SIR-Spheres microspheres may be used at any point in the patient journey, from first-line setting through to salvage therapy. ${ }^{1-5}$
The safety of SIR-Spheres microspheres and maximum tolerated dose of chemotherapy agents has been established in combination with FOLFOX, irinotecan, 5-FU/LV and FUDR regimens for colorectal cancer, as well as in monotherapy across a wide range of primary and secondary liver cancers. ${ }^{1-9}$ An extensive on-going clinical studies programme is assessing safety with other agents.
References

1. van Hazel G, Blackwell A, Anderson J et al. Randomised phase 2 trial of SIR-Spheres plus fluorouracil/leucovorin chemotherapy versus fluorouracil/leucovorin chemotherapy alone in advanced colorectal cancer. Journal of Surgical Oncology 2004; 88: 78-85.

2. Gray B, van Hazel G, Hope M et al. Randomised trial of SIR-Spheres plus chemotherapy vs chemotherapy alone for treating patients with liver metastases from primary large bowel cancer. Annals of Oncology 2001; 12: 1711-1720.

3. Kennedy A, Coldwell D, Nutting C et al. Resin ${ }^{90} \mathrm{Y}$ microsphere brachytherapy for unresectable colorectal metastases: Modern USA experience. International Journal of Radiation Oncology, Biology and Physics 2006; 65: 412-425.
4. Goldstein D, van Hazel G, Pavlakis N et al. Selective Internal Radiation Therapy (SIRT) plus systemic chemotherapy with irinotecan. A phase I dose escalation study. Journal of Clinical Oncology 2005 ASCO Annual Meeting Proceedings. 23 (16S, Part I of II): Abstract 3701

5. Sharma R, van Hazel G, Morgan B et al.

Radioembolization of liver metastases from colorectal cancer using yttrium-90 microspheres with concomitant systemic oxaliplatin, fluorouracil, and leucovorin chemotherapy. Journal of Clinical Oncology 2007; 25: 1099-1106.

6. Lau W, Ho S, Yu S et al. Salvage surgery following downstaging of unresectable hepatocellular carcinoma. Annals of Surgery 2004; 240: 299-305.
7. Hoffmann RT, Jakobs TF, Trumm C et al. Radiofrequency ablation after downstaging using selective internal radiation therapy (SIRT) - is it feasible? Radiological Society of North America (RSNA) Annual Meeting 2006; Abstract SSC03-07.

8. Lim L, Gibbs P, Yip D et al. Prospective study of treatment with selective internal radiation therapy spheres in patients with unresectable primary or secondary hepatic malignancies. Internal Medicine Journal 2005; 35: 222-227.

9. Jakobs TF, Hoffmann RT, Poepperl G et al. Mid-term results in otherwise treatment refractory primary or secondary liver confined tumours treated with selective internal radiation therapy (SIRT) using ${ }^{90} \mathrm{Yttrium}$ resinmicrospheres. European Radiology 2007; 17: 1320-1330. 